\title{
Magnetic Fields in Gamma-Ray Bursts: A Short Overview
}

\author{
Tsvi Piran \\ Racah Institute for Physics, The Hebrew University, Jerusalem, 91904 Israel \\ Theoretical Astrophysics, Caltech, Pasadena, CA 91125, USA
}

\begin{abstract}
Magnetic fields play a crucial role in the physics of Gamma-Ray Bursts (GRBs). Strong observational evidence indicates that the observed afterglow and most likely the prompt emission arise from synchrotron emission. It is possible that Poynting flux plays an important or even dominant role in the relativistic outflow from the inner engine, but like in other astronomical relativistic jets this suggestion is controversial. Finally, it is likely that magnetic fields larger than $10^{15} \mathrm{G}$ occur within GRBs' inner engines and contribute to the acceleration and collimation of the relativistic jets. I review here the GRB fireball model and discuss the role that magnetic fields play in its various components. I suggest that the early afterglow, that reflects the initial interaction of the relativistic jet with its surrounding matter is the best available tool to explore the nature of relativistic outflow in astronomical relativistic jets.
\end{abstract}

Keywords: Gamma-Ray Bursts, Magnetic Fields, Shock Waves

PACS: $98.70 . \mathrm{Rz}, 47.65 .+\mathrm{a}, 47.75 .+\mathrm{f}, 52.35$.

\section{INTRODUCTION}

More than thirty years, after the discovery of Gamma-Ray bursts (GRBs) we have now a reasonable GRB model. The model is based on the dissipation of an ultra-relativistic outflow. At first the flow is dissipated by internal shocks (or another form of internal dissipation) that produce the prompt $\gamma$-rays. Later the interaction of the flow with the circum-burst matter produces an external shock and this blast wave produces the subsequent afterglow.

The role of magnetic fields varies within GRB models from crucial to ultimate. It is generally accepted that the observed afterglow is produced by synchrotron emission $[1,2,3,4]$. Synchrotron is also the best-bet model for the prompt $\gamma$-rays emission (note however some criticism of the synchrotron model for the prompt emission [5, $6,7])$. Even within the conventional model of a baryonic relativistic outflow $[8,9,10]$ magnetic fields within the shocked regions are essential to produce this synchrotron emission. In the maximal case the whole phenomenon is magnetic. The inner engine that produces the relativistic outflow is driven by the Blandford-Znajek mechanism [11] from a magnetized black hole - accretion disk system [12, 13, 14, 15], or by a rapidly rotating highly magnetized pulsar $[16,17,18]$. The relativistic outflow is a Poynting flux $[16,17,18,15]$ and the dissipation arises due to magnetic field recombination or some other instability. Even with a baryonic outflow magnetic fields may play a dominant role in the internal engine as a possible way to power the relativistic outflow is by reconnection of $10^{15} \mathrm{G}$ magnetic fields within an accretion disk surrounding a compact object [19]. 
We can observe directly only the emitting regions where the dissipation and the emission take place. The inner engine that accelerates and collimates the relativistic outflow and the outflow itself are hidden. Both could not be observed directly and we have only indirect clues on their nature. Our inability to explore directly the inner engine and the nature of the relativistic outflow (Poynting flux or baryonic) is quite general. The same problem arises in modelling relativistic jets seen in many other astronomical objects. Specifically the relativistic jets in AGNs and galactic micro quasars are the two most similar to GRB jets. GRBs might hold the key for the resolution of the question what are these flow made of?

I begin this short review with a brief discussion of the fireball model focusing on issues most directly relevant to magnetic fields. I refer the reader to [20] for a more general and extended review and to [21] for a rather different perspective on magnetic fields in GRBs. I discuss the implication of synchrotron emission on magnetic fields within the afterglow and the prompt emission. I examine what can we learn from polarization measurements. After that I turn to the early afterglow and discuss the role of the prompt optical flash as a diagnostic of the nature of the relativistic outflow. I conclude with a short discussion of future prospects for progress on these issues.

\section{THE INTERNAL-EXTERNAL SHOCKS MODEL}

Figure 1 depicts an overall view of the internal-external shocks model. A compact source whose size is $\sim 10^{6} \mathrm{~cm}$ emits a collimated ultra-relativistic wind with a Lorentz factor of at least 100. For a long duration GRB this might take place within a collapsing star, as suggested by the collapsar model [22] and confirmed by the association of long duration GRBs with type Ic supernovae [23, 24, 25, 26]. In this case the jet has to punch a hole in the stellar envelope, whose radius is $\sim 10^{10} \mathrm{~cm}$. This envelope is not depicted in this picture.

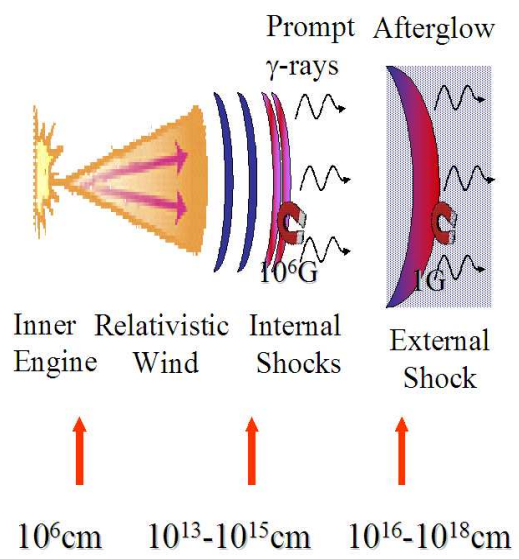

FIGURE 1. A schematic diagram of the internal-external shocks model. The magnets depict the essential magnetic fields in this model and their typical values. 
The kinetic energy of the wind is partially dissipated via internal shocks that take place around $10^{13}-10^{15} \mathrm{~cm}$. These shocks accelerate the electrons to ultra-relativistic energies (the typical Lorentz factor of an electron is $\sim 1000$ ). The electrons emit the observed prompt $\gamma$-rays via synchrotron radiation. The needed magnetic field is carried from the inner engine or is generated and amplified by the shocks.

At a distance of $\sim 10^{16} \mathrm{~cm}$ the loading of the circum-burst material on the outgoing flow becomes effective and the ejecta begins to slow down. At first a two shocks system forms. A forward shock propagating into the circum-burst matter, a reverse shock propagating into the ejecta and a contact discontinuity between the two. This is a short lived phase during which the early afterglow arises.

After the reverse shock crosses the ejecta a rarefaction wave passes and the ejecta cools down adiabatically. At this stage, at distances of $10^{16}-10^{18} \mathrm{~cm}$ from the center, the forward shock collects more and more circum-burst material. It expands adiabatically and approaches the self similar Blandford-McKee solution [27]. This is the ultrarelativistic analog of the well known Sedov-Taylor Newtonian blast wave solution. As more material accumulates the shock slows down, with the Lorentz factor decreasing like $R^{-3 / 2}$ and $t^{-3 / 8}$.

Sometime during this phase the Lorentz factor $\Gamma$, drops below $\theta^{-1}$ the opening angle of the jetted outflow. At this stage we encounter a jet break in the afterglow light curve. For $\Gamma>\theta^{-1}$ most of the emitted radiation is beamed within $\theta$ but for $\Gamma<\theta^{-1}$ some of the radiation is emitted sideways. Additionally for $\Gamma<\theta^{-1}$ the outflow expands sideways and if the expansion is rapid enough the additional collected matter causes a faster slowing down.

Eventually, around $10^{18} \mathrm{~cm}$ the blast wave collects sufficient material to slow it down so that it reaches the Newtonian transition. This takes place a few months to a year after the burst. At this stage practically all the radiation is in the radio frequencies. Later the blast wave approaches the Taylor-Sedov solution.

The magnetic field parameters in the different regimes are estimated from the observed emission, that is assumed to be synchrotron (see the discussion below). In Table 1 I compare various quantities relating to the magnetic fields in the various regimes.

TABLE 1. Magnetic parameters within GRBs: The size of the region $R$, the magnetic field $B$, the electron's Larmour radius, $R_{L} \equiv \gamma m_{e} c^{2} / e B, \lambda_{B}$ the maximal correlation length needed for jitter radiation, the skin depth $\delta \equiv c / \omega_{p}$, and the width of the emitting region (in the observer frame) $\Delta$.

\begin{tabular}{|c|c|c|c|c|c|c|c|c|}
\hline & $\mathrm{R}$ & $\mathrm{B}$ & $R_{L}$ & $\lambda_{B}$ & $\Delta$ & $R_{L} / \Delta$ & $\delta$ & $\Delta / \delta$ \\
\hline Internal Shocks & $10^{13}-10^{15} \mathrm{~cm}$ & $10^{6} \mathrm{G}$ & $1 \mathrm{~cm}$ & $10^{-3} \mathrm{~cm}$ & $10^{11} \mathrm{~cm}$ & $10^{-7}$ & $100 \mathrm{~cm}$ & $10^{9}$ \\
\hline Afterglow & $10^{16}-10^{18} \mathrm{~cm}$ & $1 \mathrm{G}$ & $10^{6} \mathrm{~cm}$ & $10^{2} \mathrm{~cm}$ & $10^{16} \mathrm{~cm}$ & $10^{-9}$ & $10^{6} \mathrm{~cm}$ & $10^{9}$ \\
\hline
\end{tabular}

An alternative model is based on a Poynting flux outflow. In this case the inner engine is magnetic and the dissipation is in the form of magnetic field recombination. General arguments suggest that the sizes of the inner engine and of the different emitting regimes should be comparable to those within the baryonic flow model. The magnetic fields are however, larger as they not only contribute to the synchrotron emission but they also carry most of the energy. Figure 2 depicts a schematic description of this model. It is also possible of course that the Poynting flux energy and the baryonic energy are comparable leading to a mixed model [28]. 


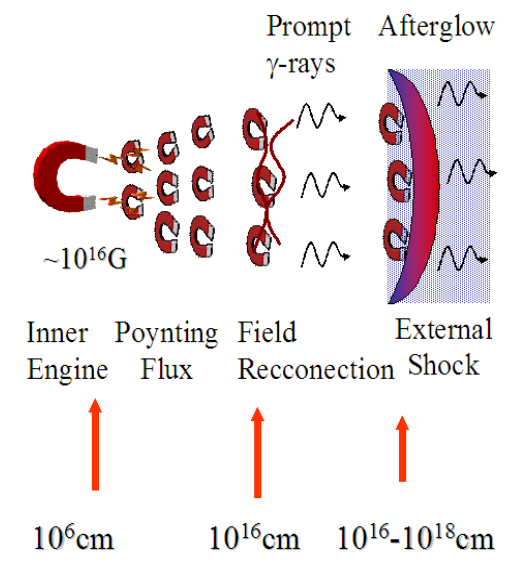

FIGURE 2. A schematic diagram of the Poynting flux model.

\section{SYNCHROTRON EMISSION IN GRBS}

The shock accelerated electrons emit, via synchrotron emission, the observed radiation. Figure 3 (from [3]) depicts the typical synchrotron spectrum for fast cooling, which is applicable during the GRB prompt phase and for slow cooling that is applicable for the afterglow. It is generally assumed that the energy densities of the relativistic electrons and of the magnetic field can be characterized by equipartition parameters, $\varepsilon_{e}$ and $\varepsilon_{B}$ that measure the ratios of these energies to the total energy. It is also assumed that the electrons energy distributed behaves like a power law with an index $-p$. Fits to the observed afterglow spectra suggest that $\varepsilon_{e} \sim 0.2, \varepsilon_{B} \sim 0.001$ and $p \sim 2.3-2.5[29,30]$.

Schematically, the spectrum is composed of four segments separated by three typical frequencies. The cooling frequency that is the synchrotron frequency of an electron that cools on a hydrodynamic time scale, the synchrotron frequency of the typical electron and the self absorption frequency (see [3] and [31] for details).

\section{Magnetic Fields in the Afterglow}

The Blandford-McKee ultra-relativistic self-similar solution combined with the synchrotron radiation model provides an excellent description of the afterglow light curves and spectra. As one can see from Table 1 the magnetic fields in this region are relatively large. The value of $1 \mathrm{G}$ is much larger than the one obtained by a simple shock compression of the intergalactic magnetic field. Even if the burst takes place within a wind that was ejected from the star prior to its explosion the magnetic fields carried out by the wind at $10^{16} \mathrm{~cm}$ would be too weak. An immediate question that arises is what is the origin of this strong magnetic field. This is particularly puzzling as the afterglow is emitted by shocked circum-burst matter whose magnetic field cannot be carried from the inner engine (as can take place in the internal shocks). The field has to be generated 

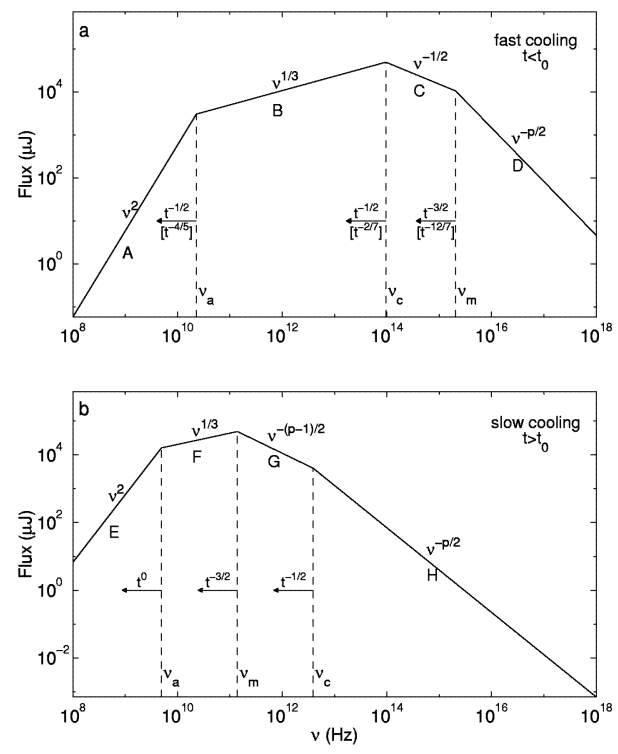

FIGURE 3. A schematic synchrotron spectrum during the fast (top) and slow (bottom) cooling phases.

in site by the shock.

Weibel [32] suggested already in 1959, that an instability leading to the growth of the magnetic field would arise whenever two flows cross each other. Kazimura et al., [33] have shown that such an instability could lead to growth of the magnetic field and to particle acceleration. As the collisionless shocks that arise in GRB afterglows are composed of two flows that cross each other Medvedev and Loeb [34] suggested that the Weibel instability is the origin of the strong magnetic fields inferred in GRB afterglows. However, Gruzinov [35] raised the concern that this magnetic field will maintain its equipartition magnitude only over a skin depth $\delta$ which for GRB afterglows is around $10^{6} \mathrm{~cm}$ - ten orders of magnitude smaller than the width of the emitting region (see table 1. While I am not aware of a simple analytic explanation of this puzzle recent numerical simulations [36, 37, 38, 39] suggest that equipartition size magnetic field remains over a region of thousands of skin depth. Moreover, the same instability accelerates particles to relativistic energies $[36,40]$, providing another ingredient of the synchrotron emission. While current simulations cannot imitate the realistic conditions they clearly suggest that the required magnetic fields can be amplified in afterglow shocks and hence in other relativistic collisionless shocks.

\section{Synchrotron Prompt Emission}

The energy density within the internal shocks region is much larger than the energy density within the afterglow, and so are the corresponding magnetic fields that can be as large as $10^{6}-10^{7} \mathrm{G}$ (depending on the exact location of the shocks and on their equipartition parameters). Here the field can be generated at the shocks (by the same Wiebel instability) or it can be carried out from the inner engine. The toroidal component 
of the field decays only as $R^{-1}$ and hence a field of $10^{14} \mathrm{G}$ at $10^{6} \mathrm{~cm}$ can easily reach $10^{6}$ $\mathrm{G}$ at $10^{14} \mathrm{~cm}$. Note that such a magnetic field is energetically subdominant and it does not require that the flow will be Poynting flux. A Poynting flux dominated flow requires at least $10^{15} \mathrm{G}$ at the inner engine and at least $10^{7} \mathrm{G}$ at $10^{14} \mathrm{~cm}$.

While synchrotron emission fits nicely the observed afterglow spectra and light curves it is not clear that it fits the observed prompt $\gamma$-ray spectrum. Specifically, the low energy part of the synchrotron spectrum behaves like $v^{1 / 3}$ (see Fig. 3) [1, 5]. However many GRBs show a steeper low energy spectrum. That is many spectra fall below the "synchrotron line of death" $[41,42,6]$. The constraint is even more severe, considering the fact that for the required high efficiency the system must be fast cooling and this requires a spectrum less steep than $v^{-1 / 2}$ [5]. These observations have led some authors $[43,7]$ to rule out synchrotron as the source of the prompt $\gamma$-ray emission. However, the low energy spectral observations of the more sensitive (in this energy band) HETE [44] do not show numerous bursts below the "lines of death". We may have to wait for better data to determine this issue.

The problems that the synchrotron model encountered in fitting the observed GRB spectra have led to the suggestions that Synchrotron-self-Compton [43, 7] and inverseCompton of an external photon field [46, 47, 48] are the emission processes. Medvedev [45] proposed that the prompt emission is produced by Jitter radiation - the analogue of synchrotron radiation in a random field [49]. Jitter radiation arises in cases that the magnetic field's correlation length $\lambda_{B}$ is smaller than the region over which an electron emits the synchrotron radiation seen by a given observer, $R_{L} / \gamma$ (see Figure 4). The spectra of Jitter radiation is more complicated than the synchrotron spectra and Medvedev suggests that it could have a low energy spectrum that is steeper than $v^{-1 / 2}$ or even than $v^{1 / 3}$. See however [50] for remarks on Medvedev's results.

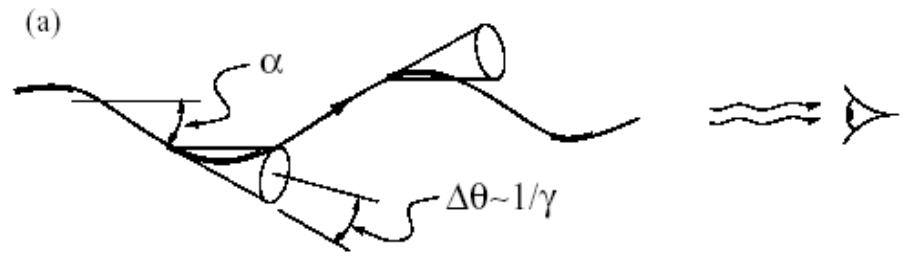

(b)

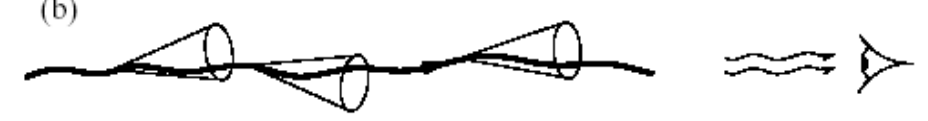

FIGURE 4. A schematic diagram of the motion of an electron in a random magnetic field and Jitter radiation (a) Emission from selected parts of the trajectory are seen by the observer. (b) Emission from the the entire trajectory is seen by the observer (see also [45]). 


\section{POLARIZATION}

\section{Afterglow Polarization}

As synchrotron emission is intrinsically polarized with a suitable geometry it would lead to polarized afterglow [51, 52, 53, 54]. In particular the required geometry could occur when we observe a relativistic jet. By now polarized optical emission has been observed from several afterglows $[55,56,57,58,59]$. Somewhat surprisingly the polarization magnitude and angle do not follow the simple predictions [52, 53, 60]. While the predicted polarization was at the level of a ten percent the observed polarization was only around a few percent. Furthermore, the jet structure suggests a specific polarization pattern for a uniform jet $[52,53]$ and another one for a universal structured jet [60]. None was observed so far. The patchy shell model in which the local geometry of the jet is dominated by hot spots [61] provides a possible explanation for the lower polarization level and the jumps in the polarization angle [62].

Granot and Taylor [63] reported recently upper limits on the polarization of GRB radio flares (the early part of the radio afterglow). Their best limit, for GRB 991216 is around $10 \%$. They point out that this upper limit already rules out a universal structure jet model with a homogenous magnetic field. More refines observations will enable us to distinguish between different jet models.

\section{Polarized Prompt Emission}

Coburn and Boggs [64] announced the discovery of very strong ( 80\%) polarization from the prompt emission of GRB 021206. It has been argued that this level of polarization shows that the magnetic filed must be uniform $[64,65]$ and that this further implies that the flow must be Poynting flux dominated [66]. However, two independent groups $[67,68]$ reanalyzed the same data and found no statistical viable indication of polarization. Additionally, synchrotron emission from a relativistic jet [69] can produce $\mathrm{a} \sim 50 \%$ polarized light even with a random magnetic field (provided that the field is within the plane of the shock). This is only slightly lower than the maximal emission from a homogenous magnetic field configuration $[65,69]$ which is around $60 \%$. Furthermore, a homogenous (toroidal) field configuration would arise in any case when the field is dragged from the source [28]. Thus, homogeneity does not necessarily imply that the flow is Poynting flux dominated. We still need a better way to distinguish between Poynting flux and baryonic outflow.

\section{THE EARLY AFTERGLOW - A DIAGNOSTIC TOOL OR THE NATURE OF THE OUTFLOW}

One of the most interesting and most vividly debated questions conceding GRBs is the nature of the relativistic outflow - Poynting flux or a baryonic outflow. One of the best ways to explore this question is by observation and analysis of the early afterglow 
FIGURE 5. A schematic diagram of the early afterglow optical and Radio light curves.

\section{$[70,71,72]$.}

The initial interaction of the relativistic outflow with the circum-burst material results in two shocks. A short lived reverse shock that propagates into the outflow and a long lived forward shock that propagates into the circum-burst material. The later eventually turns into the relativistic blast wave that produces the afterglow. Recently Nakar and Piran [70] presented a new analysis of the reverse shock emission (see also [73]). They show that a baryonic jet has a unique signature and that a combined analysis of the optical and the radio data could, therefore, enable us to explore the nature of the outflow.

The reverse shock optical emission peaks at $t_{o}$ when the reverse shock reaches the inner edge of the outflow. The first unique signature of the reverse shock is a characteristic decay of the optical flux after $t_{o}$ as $\sim t^{-2}[74,75]$. This decay arises due to the adiabatic cooling of the shocked material. The behavior is quite different at the radio. Initially, the typical synchrotron frequency, $v_{m}$ is above the observed radio frequency $v_{R}$, and below the self absorption frequency, $v_{a}$. As the shocked material cools $v_{m}$ decreases and the radio emission increases as $t^{1 / 2}$ until $v_{m}=v_{R}$. At this stage the radio flux increases like $t^{3 / 2}$ until, at $t_{R} v_{a}=v_{R}$ and from that moment on the radio emission decreases as $t^{-2}$ just like the optical. This behavior is schematically depicted in Figure 5

For baryonic outflow there is a unique relation between the time and flux of the optical peak and the time and flux of the radio peak, $t_{o}, F_{o}\left(t_{0}\right), t_{R}$ and $F_{R}\left(t_{R}\right)$ : $\left(F_{R} / F_{o}\right)\left(t_{R} / t_{o}\right)^{(p-1) / 2+1.3}=C\left(v_{o} / v_{R}\right)^{(p-1) / 2} \approx 1000$, where $p$ is the index of the electron energy distribution and $C$ is a constant of order unity. Current uncertainties put $C$ between 0.5 and 2 . By now the only burst with well determined optical early afterglow is GRB 990123. Its optical flash decayed as $t^{-2}$. The corresponding factor that relates the optical and radio peak fluxes and peak times for GRB 990123 is 1300 very close to the expected value (1000-2000). Both results indicate a baryonic outflow in this particular burst. 


\section{SOME FUTURE PROSPECTS}

Magnetic fields are clearly a key ingredient of GRBs. They are essential in the emitting regions and it may very well be that they appear in all parts of the GRB phenomenon, including the central engine and the relativistic outflow.

As Nakar \& Piran have shown $[70,71,72]$ combined measurements of the optical afterglow and the radio flare could enable us to determine the early afterglow parameters for bursts in the nearby future. Swift and several quick response ground telescopes are ideally suited for these optical observations and hopefully radio telescopes will be allocated sufficient time and quickly enough to detect the corresponding radio flares. This would hopefully shed some light on the central question - the nature of the relativistic outflow - baryonic or Poynting flux.

Observations of prompt polarization are quite unlikely as one need an extremely bright burst, and such bursts are very rare. On the other hand the quick notification of Swift may allow measurements of many optical polarizations and possibly radio flare polarizations. These could shed further light on the signature of magnetic fields and on the angular structure of GRB jets.

As for the formation of magnetic fields in collisionless shocks, the success of the synchrotron model in explaining the basic properties of GRB afterglow demonstrates that nature knows how to generate magnetic fields in collisionless shock, even if we don't understand yet how. The progress made in numerical simulations suggest that we are on the right track and indeed it is the Weibel instability that is responsible for this field amplification. This might have some implications for seeding magnetic fields elsewhere in the Universe

\section{ACKNOWLEDGMENTS}

I thank Ehud Nakar for many helpful discussions. This research was supported in part by a US-Israel BSF grant.

\section{REFERENCES}

1. J. I. Katz, Ap. J. Lett., 432, L107-L109 (1994).

2. P. Meszaros, M. J. Rees, and R. A. M. J. Wijers, Ap. J., 499, 301 (1998).

3. R. Sari, T. Piran, and R. Narayan, Ap. J. Lett., 497, L17 (1998).

4. N. M. Lloyd, and V. Petrosian, Ap. J., 543, 722-732 (2000).

5. E. Cohen, J. I. Katz, T. Piran, R. Sari, R. D. Preece, and D. L. Band, Ap. J., 488, 330 (1997).

6. R. D. Preece, M. S. Briggs, T. W. Giblin, R. S. Mallozzi, G. N. Pendleton, W. S. Paciesas, and D. L. Band, Ap. J., 581, 1248-1255 (2002).

7. B. E. Stern, and J. Poutanen, Mon. Not. RAS, 352, L35-L39 (2004).

8. A. Shemi, and T. Piran, Ap. J. Lett., 365, L55-L58 (1990).

9. B. Paczynski, Ap. J., 363, 218-226 (1990).

10. P. Mészáros, and M. J. Rees, Mon. Not. RAS, 257, 29P-31P (1992).

11. R. D. Blandford, and R. L. Znajek, Mon. Not. RAS, 179, 433-456 (1977).

12. H. K. Lee, G. E. Brown, and R. A. M. J. Wijers, Ap. J., 536, 416-419 (2000).

13. H. K. Lee, R. A. M. J. Wijers, and G. E. Brown, Phys. Rep., 325, 83-114 (2000).

14. M. H. P. M. van Putten, Phys. Rep., 345, 1-59 (2001). 
15. M. Lyutikov, and R. Blandford, ArXiv Astrophysics e-prints (2003), astro-ph/0312347.

16. V. V. Usov, Nature, 357, 472-474 (1992).

17. C. Thompson, Mon. Not. RAS, 270, 480 (1994).

18. J. I. Katz, Ap. J., 490, 633 (1997).

19. R. Narayan, B. Paczynski, and T. Piran, Ap. J. Lett., 395, L83-L86 (1992).

20. T. Piran, Rev. Mod. Phys, 76, 1143 (2004).

21. M. Lyutikov, ArXiv Astrophysics e-prints (2004), astro-ph/ 0409489.

22. A. I. MacFadyen, and S. E. Woosley, Ap. J., 524, 262-289 (1999).

23. T. J. Galama, P. M. Vreeswijk, J. van Paradijs, C. Kouveliotou, T. Augusteijn, H. Bohnhardt, J. P. Brewer, V. Doublier, J.-F. Gonzalez, B. Leibundgut, C. Lidman, O. R. Hainaut, F. Patat, J. Heise, J. in 't Zand, K. Hurley, P. J. Groot, R. G. Strom, P. A. Mazzali, K. Iwamoto, K. Nomoto, H. Umeda, T. Nakamura, T. R. Young, T. Suzuki, T. Shigeyama, T. Koshut, M. Kippen, C. Robinson, P. de Wildt, R. A. M. J. Wijers, N. Tanvir, J. Greiner, E. Pian, E. Palazzi, F. Frontera, N. Masetti, L. Nicastro, M. Feroci, E. Costa, L. Piro, B. A. Peterson, C. Tinney, B. Boyle, R. Cannon, R. Stathakis, E. Sadler, M. C. Begam, and P. Ianna, Nature, 395, 670-672 (1998).

24. J. S. Bloom, S. R. Kulkarni, S. G. Djorgovski, A. C. Eichelberger, P. Cote, J. P. Blakeslee, S. C. Odewahn, F. A. Harrison, D. A. Frail, A. V. Filippenko, D. C. Leonard, A. G. Riess, H. Spinrad, D. Stern, A. Bunker, A. Dey, B. Grossan, S. Perlmutter, R. A. Knop, I. M. Hook, and M. Feroci, Nature, 401, 453-456 (1999).

25. K. Z. Stanek, T. Matheson, P. M. Garnavich, P. Martini, P. Berlind, N. Caldwell, P. Challis, W. R. Brown, R. Schild, K. Krisciunas, M. L. Calkins, J. C. Lee, N. Hathi, R. A. Jansen, R. Windhorst, L. Echevarria, D. J. Eisenstein, B. Pindor, E. W. Olszewski, P. Harding, S. T. Holland, and D. Bersier, Ap. J. Lett., 591, L17-L20 (2003).

26. J. Hjorth, J. Sollerman, P. Møller, J. P. U. Fynbo, S. E. Woosley, C. Kouveliotou, N. R. Tanvir, J. Greiner, M. I. Andersen, A. J. Castro-Tirado, J. M. Castro Cerón, A. S. Fruchter, J. Gorosabel, P. Jakobsson, L. Kaper, S. Klose, N. Masetti, H. Pedersen, K. Pedersen, E. Pian, E. Palazzi, J. E. Rhoads, E. Rol, E. P. J. van den Heuvel, P. M. Vreeswijk, D. Watson, and R. A. M. J. Wijers, Nature, 423, 847-850 (2003).

27. R. D. Blandford, and C. F. McKee, Physics of Fluids, vol. 19, Aug. 1976, p. 1130-1138., 19, 11301138 (1976)

28. H. C. Spruit, F. Daigne, and G. Drenkhahn, Astron. \& Astrophys., 369, 694-705 (2001).

29. A. Panaitescu, and P. Kumar, Ap. J. Lett., 560, L49-L53 (2001).

30. S. A. Yost, F. A. Harrison, R. Sari, and D. A. Frail, Ap. J., 597, 459-473 (2003).

31. J. Granot, and R. Sari, Ap. J., 568, 820-829 (2002).

32. E. S. Weibel, Physical Review Letters, 2, 83-84 (1959).

33. Y. Kazimura, J. I. Sakai, T. Neubert, and S. V. Bulanov, Ap. J. Lett., 498, L183 (1998).

34. M. V. Medvedev, and A. Loeb, Ap. J., 526, 697-706 (1999).

35. A. Gruzinov, Ap. J. Lett., 563, L15-L18 (2001).

36. K.-I. Nishikawa, P. Hardee, G. Richardson, R. Preece, H. Sol, and G. J. Fishman, Ap. J., 595, 555-563 (2003).

37. C. H. Jaroschek, H. Lesch, and R. A. Treumann, Ap. J., 616, 1065-1071 (2004).

38. J. T. Frederiksen, C. B. Hededal, T. Haugbølle, and Å. Nordlund, Ap. J. Lett., 608, L13-L16 (2004).

39. M. V. Medvedev, M. Fiore, R. A. Fonseca, L. O. Silva, and W. B. Mori, Ap. J. Lett., 618, L75-L78 (2005).

40. C. B. Hededal, T. Haugbølle, J. T. Frederiksen, and A. Nordlund, Ap. J. Lett., 617, L107-L110 (2004).

41. R. D. Preece, M. S. Briggs, R. S. Mallozzi, G. N. Pendleton, W. S. Paciesas, and D. L. Band, Ap. J. Lett., 506, L23-L26 (1998).

42. R. D. Preece, M. S. Briggs, R. S. Mallozzi, G. N. Pendleton, W. S. Paciesas, and D. L. Band, Ap. J. Supp., 126, 19-36 (2000).

43. G. Ghisellini, and A. Celotti, Ap. J. Lett., 511, L93-L96 (1999).

44. C. Barraud, J.-F. Olive, J. P. Lestrade, J.-L. Atteia, K. Hurley, G. Ricker, D. Q. Lamb, N. Kawai, M. Boer, J.-P. Dezalay, G. Pizzichini, R. Vanderspek, G. Crew, J. Doty, G. Monnelly, J. Villasenor, N. Butler, A. Levine, A. Yoshida, Y. Shirasaki, T. Sakamoto, T. Tamagawa, K. Torii, M. Matsuoka, E. E. Fenimore, M. Galassi, T. Tavenner, T. Q. Donaghy, C. Graziani, and J. G. Jernigan, Astron. \& Astrophys., 400, 1021-1030 (2003). 
45. M. V. Medvedev, Ap. J., 540, 704-714 (2000).

46. A. Shemi, Mon. Not. RAS, 269, 1112 (1994).

47. N. J. Shaviv, and A. Dar, Ap. J., 447, 863 (1995).

48. D. Lazzati, E. Rossi, G. Ghisellini, and M. J. Rees, Mon. Not. RAS, 347, L1-L5 (2004).

49. I. A. Nikolaev, and V. N. Tsytovich, Physica Scripta, 20, 665-668 (1979).

50. G. D. Fleishman, ArXiv Astrophysics e-prints (2005), ast ro-ph/ 0502245.

51. A. Gruzinov, and E. Waxman, Ap. J., 511, 852-861 (1999).

52. R. Sari, Ap. J. Lett., 524, L43-L46 (1999).

53. G. Ghisellini, and D. Lazzati, Mon. Not. RAS, 309, L7-L11 (1999).

54. A. Gruzinov, Ap. J. Lett., 525, L29-L31 (1999).

55. S. Covino, D. Lazzati, G. Ghisellini, P. Saracco, S. Campana, G. Chincarini, S. di Serego, A. Cimatti, L. Vanzi, L. Pasquini, F. Haardt, G. L. Israel, L. Stella, and M. Vietri, Astron. \& Astrophys., 348, L1-L4 (1999).

56. R. A. M. J. Wijers, P. M. Vreeswijk, T. J. Galama, E. Rol, J. van Paradijs, C. Kouveliotou, T. Giblin, N. Masetti, E. Palazzi, E. Pian, F. Frontera, L. Nicastro, R. Falomo, P. Soffitta, and L. Piro, Ap. J. Lett., 523, L33-L36 (1999).

57. E. Rol, R. A. M. J. Wijers, P. M. Vreeswijk, L. Kaper, T. J. Galama, J. van Paradijs, C. Kouveliotou, N. Masetti, E. Pian, E. Palazzi, F. Frontera, and E. P. J. van den Heuvel, Ap. J., 544, 707-711 (2000).

58. J. Greiner, S. Klose, K. Reinsch, H. Martin Schmid, R. Sari, D. H. Hartmann, C. Kouveliotou, A. Rau, E. Palazzi, C. Straubmeier, B. Stecklum, S. Zharikov, G. Tovmassian, O. Bärnbantner, C. Ries, E. Jehin, A. Henden, A. A. Kaas, T. Grav, J. Hjorth, H. Pedersen, R. A. M. J. Wijers, A. Kaufer, H. Park, G. Williams, and O. Reimer, Nature, 426, 157-159 (2003).

59. D. Bersier, B. McLeod, P. M. Garnavich, M. J. Holman, T. Grav, J. Quinn, J. Kaluzny, P. M. Challis, R. G. Bower, D. J. Wilman, J. S. Heyl, S. T. Holland, V. Hradecky, S. Jha, and K. Z. Stanek, Ap. J. Lett., 583, L63-L66 (2003).

60. E. M. Rossi, D. Lazzati, J. D. Salmonson, and G. Ghisellini, Mon. Not. RAS, 354, 86-100 (2004).

61. P. Kumar, and T. Piran, Ap. J., 535, 152-157 (2000).

62. E. Nakar, and Y. Oren, Ap. J. Lett., 602, L97-L100 (2004).

63. J. Granot, and G. B. Taylor, ArXiv Astrophysics e-prints (2004), astro-ph/0 412309.

64. W. Coburn, and S. E. Boggs, Nature, 423, 415-417 (2003).

65. J. Granot, Ap. J. Lett., 596, L17-L21 (2003).

66. M. Lyutikov, V. I. Pariev, and R. D. Blandford, Ap. J., 597, 998-1009 (2003).

67. R. E. Rutledge, and D. B. Fox, Mon. Not. RAS, 350, 1288-1300 (2004).

68. C. Wigger, W. Hajdas, K. Arzner, M. Güdel, and A. Zehnder, Ap. J., 613, 1088-1100 (2004).

69. E. Nakar, T. Piran, and E. Waxman, Journal of Cosmology and Astro-Particle Physics, 10, 5 (2003).

70. E. Nakar, and T. Piran, Mon. Not. RAS, 353, 647-653 (2004).

71. E. Nakar, and T. Piran, Ap. J. Lett., 619, L147-L150 (2005).

72. E. Nakar, and T. Piran, ArXiv Astrophysics e-prints (2005), astro-ph/ 0502475.

73. B. Zhang, and S. Kobayashi, ArXiv Astrophysics e-prints (2004), astro-ph/ 0404140.

74. R. Sari, and T. Piran, Ap. J., 520, 641-649 (1999).

75. S. Kobayashi, and R. Sari, Ap. J., 551, 934-939 (2001). 